Die Zusammenführung der einzelnen Landesparteien und ihrer regionalen Ontogenese in der Arena jener Beziehungen, die ein Parteiensystem ausmachen, erfolgt in einem einführenden und einem schließenden Kapitel. Einführend zeichnet Karl Schmitt die "große Wegstrecke von der Parteidiktatur zur Parteiendemokratie" in Thüringen nach. Die Neuformierung der Parteienlandschaft stand zu Beginn im Zeichen nicht nur der „Reföderalisierung der DDR", sondern auch noch jener historisch gewachsenen, klassischen Konfliktlinien, die der SED-Staat bemerkenswerterweise nicht völlig hatte zerstäuben können. „Die Vorstellung, der DDR sei 1990 ein völlig fremdes Parteiensystem übergestülpt worden, greift also zu kurz" (S. 25). Ähnlich der Zeit kurz nach der Demokratiegründung in Westdeutschland 1945/49, traten auch in Thüringen 1989/90 alte Kontinuitäten der Lagerbindung und politisch-kulturellen Orientierung wieder zutage. Die hergebrachten Konfliktlinien Kirche versus Staat und des Klassengegensatzes übertrugen sich abermals in das Wahlverhalten, jedoch im Zeichen einer „neue[n] Zuordnung von Sozialstruktur und Parteiensystem" (S. 36): Arbeiter und kirchlich gebundene Bevölkerungsteile wandten sich der CDU zu, SED-Kader und Personengruppen, die zu DDR-Zeiten systemloyal gewesen waren, der PDS. Liberale und SPD, das zeichnete sich in Thüringen bereits früh ab, waren die Verlierer im Prozess dieser elektoralen Polarisierung.

Im Schlusskapitel (Karl Schmitt / Torsten Oppelland) wird die Frage „Gelungene Konsolidierung?" mit Rückgriff auf weitere empirische Daten der Mitgliederumfrage aufgenommen. Für die dauerhafte Festigung der Parteiorganisationen sind Menge, Motivation und Partizipation der Mitglieder elementare Voraussetzungen. Im Spiegel der Daten können die Verfasser zeigen, dass die Aktivitätsprofile variieren, teils mit dem Alter der Mitglieder (wie vor allem bei der PDS), aber auch mit der Gesamtmitgliederzahl und der Anzahl zu besetzender Mandate. Quer über alle Landesparteien hinweg ist ein langfristig rückläufiger Trend bei den Mitgliederzahlen zu beobachten. Hinsichtlich der Erosion der Mitgliederpartei ist Thüringen keine Ausnahme. Zu Recht machen die Verfasser diesen für die $\mathrm{Zu}-$ kunft des Parteienstaates kritischen Punkt zur Botschaft ihrer Schlussformel: „Auch hier gilt, dass den Parteien, je weniger Mitglieder sie haben, ihre Rekrutierungsbasis für qualifiziertes politisches Führungspersonal ebenso verloren geht wie die lebendige Verbindung zu den Interessen und Problemen der Bürger" (S. 493).

Das vorliegende Thüringer Parteienhandbuch stellt einen Meilenstein der regionalen Parteienforschung dar, der für Folgepublikationen weiterer Bundesländer, in Ost- wie Westdeutschland, hohe Maßstäbe setzt.

Everhard Holtmann

\title{
Linke und rechte Kleinparteien: wenig Neues
}

Bolay, Raphael: Erfolgreiche Linke, gescheiterte Rechte. Ursachen für die Asymmetrie des deutschen Parteiensystems, Tectum Verlag, Freiburg 2010, 135 Seiten, € 24,90.

Klassische rechtspopulistische Parteien konnten - im Unterschied zur westeuropäischen Erfolgswelle - in Deutschland landesweit kaum reüssieren und niemals in den Bundestag einziehen. Der kurzfristige Erfolg eines Ronald B. Schill im Stadtstaat Hamburg liegt bereits fast ein Jahrzehnt zurück. Im Unterschied dazu gelang es der PDS, in Ostdeutschland min- 
destens drittstärkste Kraft zu werden. Im Zuge der Bundestagswahl 2005 und in den nächsten Landtagswahlen konnte die zur „Linken“ erweiterte PDS, selbst in westdeutsche Landesparlamente einziehen. Nach den Wahlen in Baden-Württemberg und Rheinland-Pfalz muss der politologische Befund der Etablierung etwas relativiert werden, da die Partei am Parlamentseinzug scheiterte. Legt man das Links-Rechts-Schema zugrunde, kann unschwer eine Asymmetrie erkannt werden, da sich auch die nun immer stärker werdenden Grünen, als „Anti-Partei-Partei“ gestartet, dauerhaft festsetzten. Woran liegt das?

Eine an der Albert-Ludwigs-Universität Freiburg entstandene Abschlussarbeit geht dieser Frage auf den Grund, nachdem am Anfang die allgemein bekannten Theorien zur Entstehung und Etablierung von Parteien erörtert werden. Einleitend steht die bekannte Kleinparteienstudie von Manfred Rowold aus dem Jahre 1974, der meinte, rechte Herausfordererparteien hätten bessere Chancen als ihre linken Widerparts. Auch die Aussage von Lazaros Miliopoulos, 2006 im Kleinparteienhandbuch von Uwe Jun, Henry Kreikenbom und Viola Neu getätigt, es könnte ein polarisierter Parteienpluralismus entstehen, erwies sich bislang als falsch.

Erst jüngst wurde die Frage wieder diskutiert, als die Piratenpartei einigen Erfolg verbuchen konnte und die Medien im Zuge der Sarrazin-Diskussion einmal mehr über die Chancen einer neuen Rechtsaußenpartei spekulierten. Bolay identifiziert drei zentrale Ursachen für die Asymmetrie: Nachfrage, Angebot und Gelegenheitsstrukturen. Mit Blick auf die Rechtsparteien ergibt sich ein allgemein bekanntes Bild. Obwohl es durchaus ein Wählerpotential gibt - Demoskopen ermittelten Ende 201018 Prozent -, fehlt es an einem einheitlichen Angebot mit einer bekannten Führungsfigur aus dem Fernsehen oder den etablierten Parteien sowie an den Gelegenheitsstrukturen. Auf der Angebotsseite konzentriert sich Bolay stark auf die NPD, vernachlässigt dabei Gruppierungen wie „Bürger in Wut“, „Pro Köln und Deutschland“, wohl aus Aktualitätsgründen die Partei „Die Freiheit“ des Berliner Abgeordneten René Stadtkewitz. Schon diese Auflistung weist auf die Heterogenität im deutschen Rechtsaußenlager hin. Ein Geert Wilders hätte in Deutschland einen schweren Stand, da durch die deutsche Vergangenheit die Öffentlichkeit schnell alarmiert wäre. Wie der Autor herausstreicht, herrscht gegenüber der Linken weniger Empörung. Eine Rechtsaußenpartei müsste sich glaubwürdig von rechtsextremen Sektierern abgrenzen, was die Linke nicht immer macht. Sie toleriert fragwürdige Gruppierungen in den eigenen Reihen, lässt auf kommunalen Listen selbst Vertreter der DKP kandidieren. Die Ko-Parteivorsitzende Gesine Lötzsch sorgte durch einen im Januar 2011 veröffentlichten Aufsatz in der „Jungen Welt“ dann doch für eine Debatte, allerdings ohne weitreichende Wirkung. Sie reflektierte über Wege zum Kommunismus, was ihr ungewohnte, in ihren eigenen Worten „hysterische Reaktionen“ einbrachte.

Insgesamt erfährt der Leser nicht wirklich Neues. Zu sehr stützt sich der Autor auf Befunde in der Literatur. Für die Parteienforschung stellt der Band dennoch eine Bereicherung dar. Eher selten konzentrieren sich Studien auf die Kleinparteien und deren Erfolgsund Misserfolgsbedingungen. Reizvoll für die weitere Forschung bleibt die Frage nach künftigen neuen Entwicklungen in der deutschen Parteienlandschaft. Immerhin haben die Volksparteien ihren einstigen Zuspruch verloren. 